\title{
Pharmacogenomics (PGx) Patient with Mixed Levels of Actionable Variant Evidence
}

Michael J. Schuh, PharmD, MBA, FAPhA; Sheena Crosby, PharmD, BCGP

Mayo Clinic Florida

\begin{abstract}
Objective: To demonstrate the types of clinical recommendations a pharmacogenomics pharmacist may make to medical clinicians with regard to medication management to improve therapeutic outcomes based on varied levels of medical literature evidence.

Summary: This case demonstrates how a common type of patient seen in a pharmacist practice may present with a varied pharmacogenomic (PGX) profile, how they may benefit from PGx analysis, and how varying levels of medical literature evidence can be used with clinical decision making.

Conclusion: PGx testing can help avoid adverse drug reactions (ADRs) or medication inefficacy by assisting in the adjustment of current or future medication doses. It can also help predict the best medications to use or those to avoid in advance by eliminating much of the existing dosing or medication selection method of trial and error.
\end{abstract}

Key words: CPIC, CYP2C19, CYP2D6, CYP3A4, Medication therapy management, MTM, PGx, Pharmacogenomics, PharmGKB

\section{Introduction}

Pharmacogenomics (PGX) lab testing has become increasingly useful in the managing of medications in clinical practice. ${ }^{1}$ By utilizing PGx lab test results, clinicians may now more accurately determine how a patient can metabolize certain medications to assist in identifying whether a medication will not work, work as expected or cause adverse drug reactions (ADRs) that may result in increased morbidity or mortality. This testing covers genes which control descriptive P-450 enzymes or CYP enzymes, primarily located in the small intestine and liver and sometimes other genes such as HLA genes. This case study will place emphasis on CYP enzymes. CYP enzymes are phase I enzymes, responsible for the pharmacokinetic metabolism of many common medications. In the past nomenclature has been confusing since both the CYP gene and the enzyme are called the same name (i.e. CYP2D6). In addition, different labs used different terminology for phenotypes, or how we actually metabolize drugs, with the same genotype. Genotype is how we are genetically programmed to metabolize drugs that may or may not be apparent on the surface like a phenotype may demonstrate.

PGx testing is usually for multiple genes that control multiple medication metabolizing enzymes and proteins, ordered as a panel with final results provided in a report. However, clinical practice implementation has been slower and more challenging

Corresponding author: Michael J. Schuh, PharmD, MBA,

FAPhA, Assistant Professor of Pharmacy

Family Medicine, and Palliative Medicine

Mayo Clinic Florida, 4500 San Pablo Rd.

Jacksonville, FL 32224

Work: 904-953-2673, Work fax: 904-953-2274

E-mail: Schuh.michael@mayo.edu than one may expect. Some explanations for this may be differences in testing methods by labs, test selection, cost to patients and providers for testing and how to report results based on evidence available from PGx labs. In addition, clinicians require additional training in the PGx terminology of the labs and training on interpretation of results as it applies to medication selection and dosing. Other barriers to practice implementation are ability of electronic health records (EMRs) to store PGx results and how to use and triage clinically actionable results to improve care. ${ }^{2-4}$ To overcome these barriers, Mayo Clinic Florida early on vetted and aligned with a Clinical Laboratory Improvement Amendments (CLIA) certified lab to provide more complete testing for many common PGx genes with reporting consistent with the best Clinical Pharmacogenetics Implementation Consortium (CPIC) medical literature evidence available. This lab also required a health care professional to communicate results to patients. ${ }^{5-6}$ Experience dictated that the best health care professional to review results and make recommendations were medication therapy management (MTM) pharmacists. In particular, MTM pharmacists specializing in polypharmacy pharmacotherapy worked best because of their frequent use of pharmacology in their day-to-day practice. In addition, the PGx practice heavily uses PharmGKB, a database that sorts and organizes CPIC results. ${ }^{1}$ Clinical annotation levels of $P G x$ evidence range from the highest level of medical literature evidence at Level $1 \mathrm{~A}$ to the lowest at Level $4 .{ }^{6}$ (Figure I) 
Figure I

Clinical Annotation Levels of Evidence
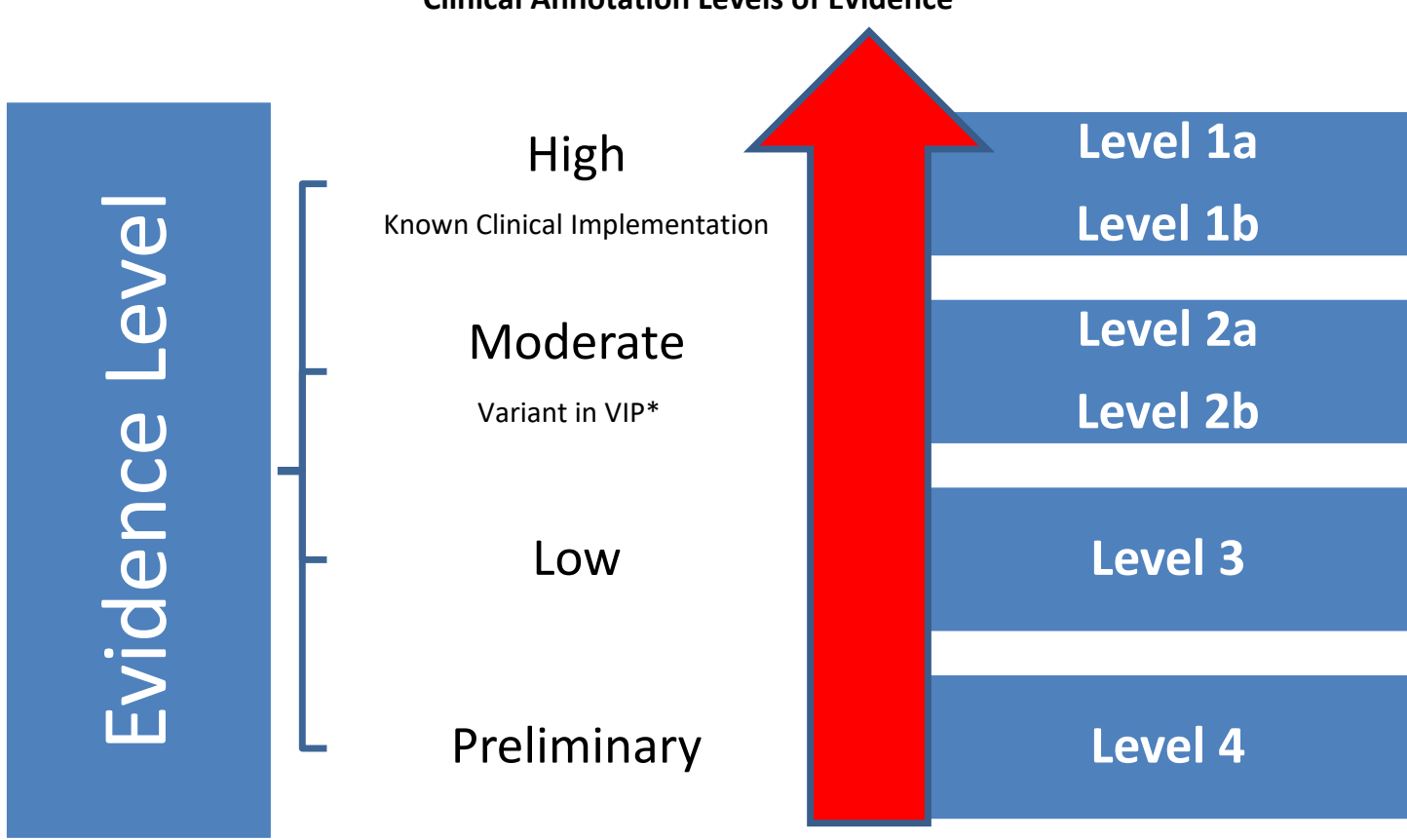

*VIP = Very Important Pharmacogene

To overcome the aforementioned PGx challenges at the Mayo Clinic Florida, the practice implemented a series of existing tools to optimize work flow. PGx patients were integrated into the work flow of pharmacotherapy patients with testing having been done weeks before to make results available at the time of the pharmacist visit. When PGx results are received by the lab they are based on available medical literature evidence, possible severity of consequences deemed actionable as clinically appropriate, then recommendations made. Until EMR alerts are fully functional for a gene, highest level evidence and actionable gene-drug interactions are listed as allergies with an explanation of why they are listed as allergies. Lower level evidence, yet possibly clinically relevant gene-drug interactions are noted in the pharmacist documentation with provider recommendations and the lowest level evidence recommendations are suggestions with softer or less definite language to match the lower level of evidence of the medical literature. Clinical rationale, with references from the medical literature is included in all actionable gene-drug interactions regarding recommendations or considerations to providers.

It was found important to reference the literature and take into consideration levels of evidence in that literature to assist in the understanding of recommendations to referring providers who were skeptical or unfamiliar with the science of PGx. Education of both providers and patients is an important part of using the science just as use of PGx should be only by those thoroughly familiar with its principles. Not having enough PGx knowledge can result in making recommendations and drug selection or dose changes exactly opposite of what is intended. This is because of some drugs having to be converted as prodrugs to the active form to work properly compared to active drugs not needing conversion by a CYP enzyme or other protein to an active form.

\section{Setting}

The Mayo Clinic Florida practice setting is a multispecialty, tertiary care clinic located in Jacksonville, Florida. The clinic offers pharmacist-provided medication therapy management (MTM), including more advanced PGx services to all patients in the clinic via referral from physicians and other providers with ordering privileges. The pharmacist PGx service sees patients from more than 20 different departments and is considered a specialty service, similar to other specialty services within the clinic. Practice is an office based practice where pharmacists see patients in a provider's office.

The two primary services performed by the PGx pharmacist are focused and comprehensive consults. PGx pharmacist visits are referred to as consults since the service is a consult or specialist service and not a longitudinal care service more associated with primary care. A focused pharmacist PGx consult is a consult, usually face to face, where the pharmacist analyzes a patient's PGx lab results and makes recommendations to the patient and provider based on those results only. This is contrary to a comprehensive pharmacist PGx consult where a comprehensive medication review is included where all Rx, OTC medications and dietary supplements are reviewed and not just medications relevant to PGx testing. With some investigational study patients, focused consults are performed via phone at the convenience of the patient. Focused PGx consults are 30 minutes; comprehensive consults are 60 minutes in length. 
The PGx service has its own calendar so all patients scheduled have had PGx testing. Scheduling capacity is 15 comprehensive, 30 focused consults per week or combination of the two types of service. OneOme (OO) and Mayo Medical Lab (MML) lab panels are used and they test for 27 and 10 genes respectively. (Table I) MML only tests for genes with the highest two levels of evidence, OO tests for genes of any listed level in PharmGKB. ${ }^{1}$ (Table I)

Table I

\begin{tabular}{|c|c|c|c|c|c|}
\hline \multicolumn{7}{|c|}{ Gene Panels } \\
\hline \multicolumn{7}{|c|}{ OneOme Lab Genes Tested } \\
\hline CYP1A2 & CYP2B6 & CYP2C9 & CYP2C19 & CYP2D6 & CYP2C19 \\
\hline CYP2C cluster & CYP3A4 & CYP3A5 & CYP4F2 & COMT & DPYD \\
\hline DRD2 & F2 & F5 & GRIK4 & HLA-A & HLA-B \\
\hline HTR2A & HTR2C & IFNL4 & NUDT15 & OPRM1 & SLC6A4 \\
\hline SLOC1B1 & TPMT & UGT1A1 & VCORC1 & Source: OneOme ${ }^{13}$ \\
\hline \multicolumn{7}{|c|}{ Mayo Medical Lab Genes Tested } \\
\hline CYP1A2 & CYP2C cluster* & CYP2C9 & CYP2C19 & CYP2D6 & SLOC1B1 \\
\hline CYP3A4 & CYP3A5 & CYP4F2 & VCORC1 & Source: Mayo Medical Labs ${ }^{14}$ \\
\hline
\end{tabular}

${ }^{*}$ CYP2C cluster is referred to as rs12777823 at Mayo Medical Labs

\section{Case Report}

Our subject is a 49 year-old white female with a past medical history of renal cell carcinoma, post coronary stent, coronary artery disease (CAD), hyperlipidemia, essential hypertension and major depression. She did not obtain depression symptom relief with lower sertraline dosing so her dose had been escalated over time. At the consult the patient reported symptoms of fatigue and tachycardia even while taking a beta blocker, leg cramping, nausea, GI cramping, myoclonic jerks and frequent diaphoresis. She was referred as a PGx investigational study patient from urology where she was being treated for her renal cell carcinoma. Urology was investigating PGx relevance to urology medications. All PGx study patients were referred to the PGx pharmacist for a focused PGx consult. Relevant medications to her PGx testing and recommendations are included in Table II.

\section{Discussion}

PGx test results yielded multiple pharmacist recommendations at varied levels of evidence. (Table II) The starred numbers in Table II represent the allele or form of a gene from each parent divided by a forward slash.

Table II

\begin{tabular}{|c|c|c|c|c|c|c|}
\hline \multicolumn{7}{|c|}{ Pharmacogenomics (PGx) Recommendation Summary } \\
\hline Drug & Daily Dose/Indication & Gene & Genotype & Phenotype & Recommendation & Evidence Level* \\
\hline tClopidogrel & $\begin{array}{c}75 \mathrm{mg} \\
\text { Once daily } \\
\text { Cardiac stent }\end{array}$ & CYP2C19 & $* 2 / * 2$ & Poor & $\begin{array}{l}\text { Change } \\
\text { clopidogrel to } \\
\text { prasugrel or } \\
\text { ticagrelor }\end{array}$ & $1 \mathrm{~A}$ \\
\hline Sertraline & $\begin{array}{c}100 \mathrm{mg} \\
\text { Twice daily } \\
\text { Depression }\end{array}$ & CYP2C19 & $* 2 / * 2$ & Poor & $\begin{array}{l}\text { Change sertraline } \\
\text { to another } \\
\text { antidepressant }\end{array}$ & $1 \mathrm{~A}$ \\
\hline Pantoprazole & $\begin{array}{c}40 \mathrm{mg} \\
\text { Once daily } \\
\text { Gastroesophageal } \\
\text { Reflux Disease (GERD) }\end{array}$ & CYP2C19 & $* 2 / * 2$ & Poor & $\begin{array}{c}\text { Consider trial } \\
\text { without or lower } \\
\text { dose }\end{array}$ & 3 \\
\hline Carvedilol & $\begin{array}{c}3.125 \mathrm{mg} \\
\text { Twice daily } \\
\text { Essential Hypertension }\end{array}$ & CYP2D6 & $(* 2 \mathrm{~A} / * 9) \times \mathrm{N}$ & Rapid & $\begin{array}{c}\text { Consider higher } \\
\text { dose }\end{array}$ & $\begin{array}{c}\text { No } \\
\text { recommendation } \\
\text { for }(* 2 \mathrm{~A} / * 9) \times N \\
\text { alleles }\end{array}$ \\
\hline Atorvastatin & $\begin{array}{c}80 \mathrm{mg} \\
\text { Once daily } \\
\text { Hyperlipidemia }\end{array}$ & CYP3A4 & $* 1 / * 1$ & $\begin{array}{l}\text { Extensive } \\
\text { (normal) }\end{array}$ & $\begin{array}{c}\text { No } \\
\text { recommendation }\end{array}$ & 3 \\
\hline $\begin{array}{l}\text { Acutely action } \\
\text { *Reference: } \mathrm{Ph}\end{array}$ & $\begin{array}{l}\text { inding } \\
\mathrm{KB}^{1} \text { (Figure I) }\end{array}$ & & & & & \\
\hline
\end{tabular}


Notably the patient's results indicated she was phenotypically a poor CYP2C19 metabolizer and a rapid CYP2D6 metabolizer. Clopidogrel, sertraline and pantoprazole are all CYP2C19 substrates while carvedilol is primarily a CYP2C9 and CYP2D6 substrate. Atorvastatin is a CYP3A4 substrate.

Sertraline and pantoprazole are active agents so poor CYP2C19 metabolizers would have little to no function of the CYP2C19 gene resulting in buildup of active drug, higher exposure and increased risk of ADRs. Patient reported classic symptoms of transient serotonin toxicity and was on a high dose of sertraline. When possible cardiovascular (CV) ADRs such as Q-T prolongation and past CV medical history were considered along with her PGx lab result as a poor CYP2C19 metabolizer, recommendation was made to change antidepressants. ${ }^{7-8}$ (Table II) Her gastroesophageal reflux disease (GERD) has been controlled and she was on a standard dose of pantoprazole. Since she may have higher exposure to pantoprazole as a poor CYP2C19 metabolizer, a lower dose of pantoprazole was suggested if she had to be taking the agent for an extended period of time to decrease possible ADRs of osteopenia/osteoporosis, renal dysfunction and lower dietary mineral or B-12 absorption. ${ }^{9-12}$ (Table II)

Conversely, clopidogrel is a prodrug and must be converted to the active form via CYP2C19 for it to irreversibly bind platelets and prevent clotting post-stent placement. ${ }^{6}$ Therefore, the patient, even with clopidogrel treatment, was still at risk for clotting and myocardial infarction (MI) because clopidogrel would not be converted to the active form and be ineffective as a poor CYP2C19 metabolizer. The clopidogrel gene-drug interaction was considered actionable because a clot or another MI was a possibility at any time. Recommendation was made to change clopidogrel to prasugrel or ticagrelor. ${ }^{6}$ On an actionable issue, the clinic referring physician to the PGx service is notified through the EMR inbox and the patient is advised to speak with the prescribing physician as soon as possible regarding the finding during the consult. Mayo Clinic physicians are aware of findings faster than referrals outside the clinic because of a shared EMR where documentation notes are posted in real time. For patients referred from afar outside the clinic, it may take longer because the patient is contacting their provider.

Carvedilol and CYP2D6 status is unclear. Though some beta blockers such as metoprolol are primarily metabolized by CYP2D6, carvedilol is metabolized by CYP2C9 and CYP2D6. ${ }^{6}$ Patient is a rapid metabolizer of CYP2D6 and extensive (normal) metabolizer of CYP2C9. Recommendation to consider a higher dose was based on observed tachycardia in this patient and seeing a lower-than-target dose of $25 \mathrm{mg}$ twice daily. In addition, the current $3.125 \mathrm{mg}$ twice daily dose with a CYP2D6 rapid metabolizer may create less exposure to the drug, resulting in her tachycardia. One could argue the tachycardia was a result of higher exposure and high dose of the sertraline, and the tachycardia resulted from high serotonin levels.
Because the patient was an extensive or normal metabolizer of CYP3A4 and stable on her atorvastatin, no recommendations were made.

\section{Conclusion}

Strength of recommendations can be made based on available medical literature evidence of PGx testing to assist with clinical decision making. For the same gene, different levels of evidence can be found for lab results associated with the different medications a patient takes resulting in different clinical implications. When using PGx testing with multiple levels of literature evidence, more medication recommendations can be made. In addition, one can more accurately select medications or make medication therapy changes that may result in better patient clinical outcomes versus the trial and error method.

In this case, potentially fatal consequences were avoided by identifying ineffective therapy in a patient without the ability to convert clopidogrel to its active form, possibly resulting in clot formation post-stent placement. Patient was recommended to take a more effective agent such as prasugrel or ticagrelor. Multiple, chronic ADRs, possibly a result of too much serotonin, could be eliminated by changing sertraline to an antidepressant not metabolized by CYP2C19. Potential dose adjustments were identified to decrease potential ADRs in the case of pantoprazole and increase efficacy in the case of carvedilol.

Many areas of medicine may benefit from PGx testing and interpretation. PGx testing can help avoid ADRs or medication inefficacy by assisting in the adjustment of current or future new medications or medication doses. It can also help predict the best medications to use or those to avoid in advance by eliminating much of the existing method of trial and error.

\begin{abstract}
Abbreviations: $A D R=$ Adverse drug reaction, $C A D=$ coronary artery disease, $\mathrm{CLIA}=$ Clinical Laboratory Improvement Amendments, $\mathrm{CPIC}=$ Clinical Pharmacogenetics Implementation Consortium CV = Cardiovascular CYP = Cytochrome P450 enzyme, EMR = electronic medical record, $\mathrm{MTM}=$ medication therapy management, $\mathrm{PGx}=$ Pharmacogenomics, OTC $=$ Over the counter, PharmGKB = The Pharmacogenomics Database
\end{abstract}

Conflicts of interest: None 


\section{References}

1. M. Whirl-Carrillo, E.M. McDonagh, J. M. Hebert, L. Gong, K. Sangkuhl, C.F. Thorn, R.B. Altman and T.E. Klein. "Pharmacogenomics Knowledge for Personalized Medicine" Clinical Pharmacology \& Therapeutics (2012);92(4):414-17.

2. Pharmacogenomics: Preemptive Testing Decisions Reach across the Care Team. Author: Kandace Schuft, PharmD. Available from: https://www.wolterskluwercdi.com/blog/pharmacog enomics-preemptive-testing-decisions/. Accessed February 15, 2019.

3. Arwood MJ, Chumnumwat $S$, Cavallari LH, Nutescu EA, Duarte JD. Implementing Pharmacogenomics at Your Institution: Establishment and Overcoming Implementation Challenges. Clin Transl Sci. 2016 Oct;9(5):233-45.

4. Moyer AM, Caraballo PJ. The Challenges of implementing pharmacogenomic testing in the clinic. Expert Review of Pharmacoeconomics \& Outcomes Research. 2017;17( 6):567-77. Available from: https://tandfonline.com/doi/full/10.1080/14737167. 2017.1385395?scroll=top\&needAccess=true. Accessed February 15, 2019.

5. Centers for Disease Control and Prevention (CDC), Clinical Laboratory Improvement Amendments. Available from: https://wwwn.cdc.gov/clia/resources/waivedtests/de fault.aspx. Accessed February 15, 2019.
6. Clinical Pharmacogenetics Implementation Consortium (CPIC). Available from: https://cpicpgx.org/. Accessed February 15, 2019.

7. Product Information: ZOLOFT(R) oral tablets, concentrate, sertraline hydrochloride oral tablets, concentrate. Pfizer Inc., New York, NY, 2009.

8. Rochester MP, Kane AM, Linnebur SA, Fixen DR. Evaluating the risk of QTc prolongation associated with antidepressant use in older adults: a review of the evidence. Ther Adv Drug Saf 2018 Jun;9(6):297-08

9. Targownik LE, Lix LM, Metge CJ, et al: Use of proton pump inhibitors and risk of osteoporosis-related fractures. CMAJ 2008;179(4):319-26.

10. Lazarus B, Chen Y, Wilson FP, et al: Proton pump inhibitor use and the risk of chronic kidney disease. JAMA Intern Med 2016;176(2):238-46.

11. Product Information: PROTONIX(R) delayed-release oral tablets, suspension, pantoprazole sodium delayed-release oral tablets, suspension. Wyeth Pharmaceuticals, Inc, Philadelphia, PA, 2008.

12. Cundy $T$ \& Dissanayake A: Severe hypomagnesaemia in long-term users of proton-pump inhibitors. Clin Endocrinol (Oxf) 2008;69(2):338-41.

13. OneOme. Available from: https://oneome.com/. Accessed December 9, 2019.

14. Mayo Clinic Laboratories. Available from: https://www.mayocliniclabs.com/testcatalog/Overview/65566. Accessed December 9, 2019. 